\title{
Investigation of the Burnout Stages Experienced by Nurses Working in Hospitals and Related Factors
}

\author{
Naomi Sumi ${ }^{*}$, Yuko Yoshida ${ }^{1}$, Naotaka Sugimura², Rika Yano ${ }^{1}$ \\ ${ }^{1}$ Faculty of Health Sciences, Hokkaido University, Sapporo, Japan \\ ${ }^{2}$ Graduate School of Health Sciences, Hokkaido University, Sapporo, Japan \\ Email: *nsumi@hs.hokudai.ac.jp
}

How to cite this paper: Sumi, N., Yoshida, Y., Sugimura, N. and Yano, R. (2018) Experienced by Nurses Working in Hospitals and Related Factors. Open Journal of Nursing, 8, 257-271.

https://doi.org/10.4236/ojn.2018.84022

Received: March 21, 2018

Accepted: April 27, 2018

Published: April 30, 2018

Copyright (c) 2018 by authors and Scientific Research Publishing Inc. This work is licensed under the Creative Commons Attribution International License (CC BY 4.0).

http://creativecommons.org/licenses/by/4.0/ (c) (i) Open Access

\begin{abstract}
Background: The purpose of this study was to elucidate the stages of nurse burnout in hospitals (their states of emotional exhaustion and depersonalization) as well as factors related to the nurses personal characteristics and coping behaviors in order to obtain suggestions for stress and health management strategies that nurses themselves can employ. Methods: We conducted a cross-sectional study using anonymous self-administered questionnaires. The subjects were nurses employed at public hospitals with 500 beds in 3 cities in Hokkaido, Japan. The questionnaire items for burnout were those included on the Japanese Maslach Burnout Inventory (MBI), which are designed to ascertain the subjects' emotional exhaustion, depersonalization, and sense of personal accomplishment. In addition, we used 11 items related to physical and mental stress responses on the Stress Coping Inventory and the Brief Job Stress Questionnaire to determine factors such as the nurses' basic attributes, whether or not they worked night shifts, and their overtime hours, etc. Results: We received responses from 1461 hospital nurses. Those whose scores for both emotional exhaustion and depersonalization were the mean or above were $40.2 \%$ of those in their twenties, $35.8 \%$ of those in their thirties, and $26.8 \%$ of those in their forties or older. The results of logistic regression analysis indicated that among those in the group that scored high for both emotional exhaustion and depersonalization, the influential factors were stress score (odds ratio 1.889), twenties age group (odds ratio 1.982), thirties age group (odds ratio 1.720), coping behavior: avoidance-focused behaviors (odds ratio 1.140), and engaged in childcare (odds ratio 0.487). Similarly, when looked at by age group, being in the twenties age group and having a spouse were influential factors (odds ratio 3.114 - 4.171). Conclusion: The results of this study elucidated the fact that for hospital nurses, the burnout stage, age,
\end{abstract}


state of physical and mental stress response, and coping behavior can be effectively used as predictive indices.

\section{Keywords}

Burnout, Coping Behavior, Hospital Nurses, Burnout, Related Factors

\section{Introduction}

Although the job separation rate for Japanese nurses is on a declining trend, in general it hovers around $11 \%$ and is $7.5 \%$ for new nurses [1]. The most commonly cited reason for nurses leaving their job is "female life events" such as childbirth and childcare, followed by physical health problems and psychological health problems [2]. Previous studies have reported that the causes for job separation among nurses include the burden of the responsibilities of the job, the number of night shifts and the length of the overtime, overwork, and other factors related to the work environment, as well as personal factors such as age and childcare [3]. In particular, those in their twenties and thirties who are at the stage during which they are building their careers as nurses are also at the age when they are experiencing major life events such as marriage and childbirth that make it difficult to continue working. Furthermore, mid-career nurses in their late twenties and thirties who are involved in providing training to novice nurses have high job demands, which increase their physical and psychological fatigue, and as a result is thought to lead to job separation among mid-career nurses [3] [4]. Maslach (1981) defined "burnout" as a "syndrome of emotional exhaustion and cynicism that occurs frequently among individuals who do 'people-work' of some kind". A key aspect of the burnout syndrome is increased feelings of emotional exhaustion [5]. Research on burnout has identified the factors related to nurse burnout as age, social support, relations with other hospital nurses, job satisfaction, and ability to cope with stress [3]. Burnout as defined by Maslach is comprised of the 3 factors of emotional exhaustion, depersonalization, and decline in personal accomplishment [5]. Although the 2 factors of emotional exhaustion and depersonalization are strongly correlated, there is said to be a weaker relationship between decline in personal accomplishment and the other 2 factors. A variety of studies have been done on the process of burnout by which emotional exhaustion leads to depersonalization. In all analyses, however, it is clear that emotional exhaustion is the central concept of burnout [6]. Previous studies of burnout among nurses and physical and psychological health suggest that chronic fatigue, depression, and other symptoms are correlated to burnout [7] [8] [9]. Early intervention into nurse burnout can prevent job separation among nurses and therefore the issue of nurses maintaining and improving their own physical and mental health is an important issue that can lead to improved quality of healthcare. 
Since 2015, in Japan, Industrial Safety and Health Act has required employers with at least 50 employees to provide worksite stress checks [10]. In addition, in 2013 the Japanese Nursing Association released its guidelines on night shifts and shift work [11], which includes educational contents related to sleepiness during nurses' shift work and outlines guidelines regarding the taking of breaks. These efforts not only reflects the importance of aspects such as work management of nurses and organizational management of hospitals, but also the importance of nurses themselves making efforts to manage stress and their own physical and mental health in order to prevent burnout. Therefore, the purpose of this study was to elucidate the stages of nurse burnout in hospitals (their states of emotional exhaustion and depersonalization) as well as factors related to the nurses themselves in order to obtain suggestions for stress and health management strategies that nurses themselves can employ.

\section{Objective of the Study}

The purpose of this study was to elucidate the stages of nurse burnout in hospitals (their states of emotional exhaustion and depersonalization) as well as factors related to the nurses themselves.

\section{Methodology}

The subjects were nurses who work in public hospitals with 500 beds in the major cities and regional cities of Hokkaido. Since their normal work schedules, including night shift work, may differ, directors and deputy directors of nursing departments as well as head nurses were excluded. All outpatient and inpatient wards were included among the subjects. The survey period was from December 2015 to January 2016. The survey was an anonymous, self-administered drop-off survey. The survey description and questionnaire forms were distributed to the subjects by the nursing departments of the participating hospitals and they were recovered by the researcher from each hospital. The subjects were also guaranteed that their participation in the survey would be of their own free will. Consent was assumed when the subjects returned their completed questionnaire forms to the researcher.

Survey items were as follows;

1) Burnout: Burnout was defined as "A long-term stress response that occurs as a result of experiencing long-term and repeated stress." We used the Japanese Burnout Scale created by Kubo that was based on the Maslach Burnout Inventory (MBI) of Maslach \& Jackson [6]. The reliability and validity of this scale has been verified. The scale consists of 17 items and 3 sub-scales (emotional exhaustion, depersonalization, and decline in personal accomplishment). Responses to each sub-scale are organized as a 5-step Likert scale, with a) Never, b) Rarely, c) Sometimes, d) Often, and e) Always. The total score for each sub-scale are then totaled. Scores are as follows: Emotional exhaustion is 5 to 25 points, and depersonalization and decline in personal accomplishment are 6 to 30 points. The 
scores for the sub-scales are then divided by the number of items. As there are no scoring criteria for this scale, a subjective assessment is made based on the variations in score among the respondents. Permission to use this scale was obtained from the developer prior to the start of this study.

2) Physical and mental stress state: We utilized the "physical and mental stress response" question items on the Workplace Stress Simple Questionnaire that was examined by the Ministry of Health, Labour and Welfare group (representative: Terukazu Shimomitsu) [10]. The following were the 11 item included: No appetite, excited, anxious, listless, blue, bothersome, can't calm down, can't sleep, feel severely tired, exhausted, depressed. They were scored as either 1: Yes or 0: No and the total was used as the "stress score" (score range: 0 - 11).

3) Stress coping: We used the Coping Scale developed by Ozeki et al. (1993) [12]. This scale measures the stress response, stressors, and coping. It is composed of 14 items organized into 3 sub-scales: Problems (5 items), emotions (3 items), and avoidance/escape (6 items). The problems-related items focus on behaviors that directly contribute to problem-solving, such as gathering information and re-examination. The emotion-related items focus on emotional responses triggered by stressors and behaviors that regulate the switching of attention and feelings. Avoidance/escape-related items are behaviors that lead to fleeing from unpleasant events or negative interpretations. The questionnaire first asks respondents to describe "the most stressful recent experience" and then asks them to rate the frequency of the psychological effort used to make decisions regarding how to reduce the stress response triggered by these stressors on a 4-step scale (ranging from 0: Never to 3: Always).

4) Nurses' basic attributes: Age, sex, marital status, engaged in childcare, engaged in nursing care, number of years employed at their hospital, work schedule and taking of annual paid leave, and monthly hours of overtime.

\subsection{Analytical Methods}

The nurses' characteristics, burnout scores, stress scores, and coping scores were calculated as the fundamental statistics. Since decline in personal accomplishment has a weaker relation to burnout than the other 2 factors, only the 2 sub-items of emotional exhaustion and depersonalization were used in analysis as the central concepts of burnout. In order to investigate the relation of the 2 sub-scales of burnout and personal attributes to the stress scores and coping, we used the independent t-test and one-way analysis of variance. We also divided the burnout sub-scales of emotional exhaustion and depersonalization into 2 groups based on means scores (High-score group and Low-score group). We considered cases in which both items were in the High-score group as the "Emotion/depersonalization group." Those that had low emotional exhaustion scores but high depersonalization scores were considered the "Depersonalization group." Those that had high emotional exhaustion scores and low depersonalization scores were considered the "Emotion group." Finally, those who had low 
scores in both sub-scales were considered the "Low-score group." Coping scores were also handled similarly using a cutoff for the mean scores for the Problem, Emotion, and Avoidance/Escape items in order to divide the subjects into a High-score and a Low-score group. In order to investigate the relation of the 4 types of burnout and the nurses' characteristics with physical/mental stress and stress coping, we performed multiple logistic regression analysis with the 4 types of burnout as the dependent variable and the stress scores, coping scores, and nurses' attributes as the independent variables. Analysis was performed using SPSS23.0v.

\subsection{Ethical Considerations}

This survey was conducted with the approval of the Institutional Review Board of the Graduate School of Health Sciences, Hokkaido University and the Institutional Review Boards of the surveyed facilities. The facilities were provided with an overview of the survey and the ethical considerations in writing. The questionnaires were distributed to the individual subjects by the nursing departments at each facility and were recovered by the researcher later.

\section{Results}

\subsection{Characteristics of the Subjects}

A total of 1890 survey forms were distributed and 1509 were recovered. The 1461 forms that did not have invalid or missing responses were used for the purpose of analysis (recovery rate: $79.8 \%$, effective recovery rate: $96.8 \%$ ). The subjects were comprised of 1335 women (91.8\%), 610 subjects were in their twenties (41.8\%), 452 subjects were in their thirties (30.9\%), and 399 subjects were in their forties (27.3\%). Five hundred two subjects were married (34.6\%), 421 were raising children at the time of the survey $(29.0 \%)$, and 120 were engaged in nursing care $(8.2 \%)$. Three hundred five subjects were receiving treatment as outpatients for some reason $(20.9 \%)$. The mean number of years of experience as a nurse was $11.6 \pm 9.0$ years. Our investigation of their work schedules indicated that 296 worked the normal day shift (20.4\%) and 1125 worked other shifts $(76.3 \%)$. The number of hours of overtime per month were as follows: 71 subjects reported they worked no overtime $(4.9 \%)$, while most subjects (703 subjects; $48.9 \%$ ) reported working under 10 hours of overtime, followed by 444 subjects who reported working 10 - 20 hours of overtime (30.9\%). Analysis of the geographical location of the subjects indicated that 1074 lived in cities $(73.5 \%)$ and 387 lived in regional cities (26.5\%) in Table 1.

\subsection{Burnout, Coping, Cumulative Fatigue, and Stress Score}

The mean scores for the 3 burnout sub-scales were as follows: Emotional exhaustion was $3.4 \pm 0.9$, depersonalization was $2.1 \pm 0.8$, and decline in sense of personal accomplishment was $3.7 \pm 0.7$. Cronbach's alpha was 0.829 (5 items), 0.852 (6 items), and 0.774 (6 items). Mean coping scores were as follows: Problems 
Table 1. Basic attributes of nurses.

\begin{tabular}{|c|c|c|c|}
\hline \multicolumn{4}{|c|}{$\mathrm{N}=1461$} \\
\hline & Item & $\mathrm{N}$ & $\%$ \\
\hline \multirow[t]{2}{*}{$\operatorname{Sex}(a)$} & Female & 1335 & 8.2 \\
\hline & Male & 119 & 91.8 \\
\hline \multirow[t]{3}{*}{ Age group } & $20 \mathrm{~s}$ & 610 & 41.8 \\
\hline & $30 \mathrm{~s}$ & 452 & 30.9 \\
\hline & 40 s and older & 399 & 27.3 \\
\hline \multirow[t]{2}{*}{ Marital status (a) } & Unmarried & 950 & 65.4 \\
\hline & Married & 502 & 34.6 \\
\hline \multirow[t]{2}{*}{ Engaged in childcare (a) } & No & 1031 & 71.0 \\
\hline & Yes & 421 & 29.0 \\
\hline \multirow[t]{2}{*}{ Engaged in nursing care (a) } & No & 1338 & 91.8 \\
\hline & Yes & 120 & 8.2 \\
\hline Years of experience as a nurse & $11.6 \pm 9.0$ & & \\
\hline No. of years at present position & $3.7 \pm 3.5$ & & \\
\hline \multirow[t]{3}{*}{ Work schedule } & Day shift & 296 & 20.4 \\
\hline & Other shifts & 1125 & 78.4 \\
\hline & Other & 20 & 1.2 \\
\hline \multirow{6}{*}{$\begin{array}{l}\text { No. of overtime hours worked in the previous } \\
\text { month (a) }\end{array}$} & None & 71 & 4.9 \\
\hline & Under 10 & 703 & 48.9 \\
\hline & 10 to under 20 & 444 & 30.9 \\
\hline & 20 to under 30 & 145 & 10.1 \\
\hline & 30 to under 40 & 52 & 3.6 \\
\hline & 40 or more & 24 & 1.7 \\
\hline \multirow{6}{*}{$\begin{array}{l}\text { No. of paid vacation days taken during the } \\
\text { previous fiscal year (a) }\end{array}$} & 0 to under 10 & 932 & 65.8 \\
\hline & 10 to under 20 & 448 & 31.6 \\
\hline & 20 to under 30 & 27 & 1.9 \\
\hline & 30 to under 40 & 4 & 0.3 \\
\hline & 40 to under 50 & 4 & 0.3 \\
\hline & 50 or more & 2 & 0.1 \\
\hline \multirow[t]{2}{*}{ Receiving outpatient care (a) } & No & 1154 & 79.1 \\
\hline & Yes & 305 & 20.9 \\
\hline \multirow[t]{2}{*}{ Location of hospital } & Major city & 1074 & 73.5 \\
\hline & Regional city & 387 & 26.5 \\
\hline
\end{tabular}

(a): Indicates the frequency and rate of the respondents.

was $7.1 \pm 2.9$, Emotions was $4.5 \pm 2.0$, and Avoidance/Escape was $8.7 \pm 3.2$. Cronbach's alpha was 0.670 (5 items), 0.564 ( 3 items), and 0.696 (6 items). The 
state of physical and mental stress was calculated as the stress score, and the mean figure was $5.1 \pm 2.9$ (range: 0 - 11 points). The Cronbach's alpha for the stress score was 0.821 (11 items; Table 2).

Analysis of the burnout categories indicated that 514 subjects (35.2\%) were in the "Emotion/Depersonalization Group" in which both emotional exhaustion and depersonalization were high, 129 subjects (8.8\%) were in the "Depersonalization Group" in which emotional exhaustion was low but depersonalization was high, 303 subjects (20.7\%) were in the "Emotion Group" in which emotional exhaustion was high but depersonalization was low, and 515 subjects (35.2\%) were in the "Low Group" in which both emotional exhaustion and depersonalization were low. Analysis of the data by age group indicated that in the Emotion/Depersonalization Group 40.2\% were in their twenties, 35.8\% were in their thirties, and $26.8 \%$ were in their forties. However, in the Low Group $28.6 \%$ were in their twenties, $35.6 \%$ were in their thirties, and $45.1 \%$ were in their forties. Analysis by geographical region indicated that more nurses who were in their twenties and in the Emotion/Depersonalization Group were located in major cities rather than regional cities (major cities:regional cities $=43.6 \%: 39.4 \%$ ). More of those in their thirties were located in regional cities than in major cities (major cities:regional cities $=25.9 \%: 39.3 \%$; Table 3 ). Analysis of stress score distributions by burnout group indicated that those in the Low Group tended to have low scores around 2. Many of those in the Emotion Group had moderate scores of 5 - 7, and many of those in the Depersonalization Group had scores of 6, although overall there was very little score dispersion. Most of those in the Emotion/Depersonalization Group had scores of 9, and compared to the other groups there were many in this group that had scores of 10 - 11 (Figure 1).

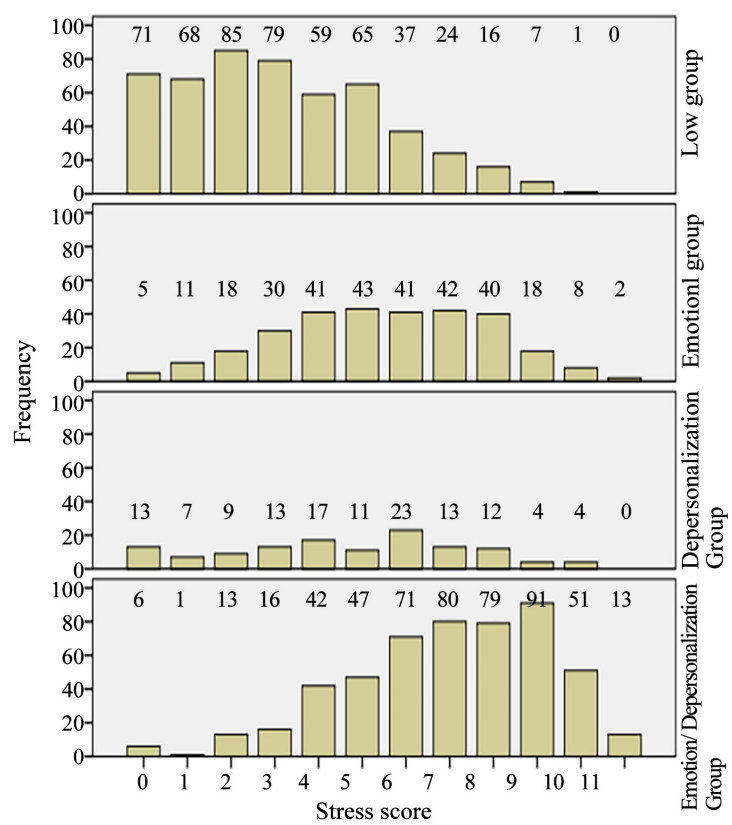

Figure 1. Distribution of stress scores throughout the 4 burnout groups. 
Table 2. Nurses' state of burnout, coping, and physical/mental stress.

\begin{tabular}{cc}
\hline & $\mathrm{N}=1461$ \\
\hline Burnout score & mean \pm SD \\
Emotional exhaustion & $3.4 \pm 0.9$ \\
Depersonalization & $2.1 \pm 0.8$ \\
Decline in sense of personal accomplishment & $3.7 \pm 0.7$ \\
Coping score & $7.1 \pm 2.9$ \\
Problem-focused & $4.5 \pm 2.0$ \\
Emotion-focused & $8.7 \pm 3.2$ \\
Avoidance-focused & $5.1 \pm 2.9$ \\
\hline
\end{tabular}

Stress score: Total score of 11 items that indicate the state of physical and mental stress (No appetite, become excited, have anxiety, feel listless, feel blue, feel bothersome, cannot calm down, cannot sleep, feel severely tired, feel exhausted, feel depressed).

Table 3. Comparison of the distribution of burnout categories by different regions.

\begin{tabular}{|c|c|c|c|c|c|c|c|c|c|c|}
\hline \multicolumn{11}{|c|}{4 Types of Burnout } \\
\hline & & \multicolumn{2}{|c|}{$\begin{array}{l}\text { Low } \\
\text { Group }\end{array}$} & \multicolumn{2}{|c|}{$\begin{array}{l}\text { Emotion } \\
\text { Group }\end{array}$} & \multicolumn{2}{|c|}{$\begin{array}{l}\text { Depersonalization } \\
\text { Group }\end{array}$} & \multicolumn{2}{|c|}{$\begin{array}{c}\text { Emotion/Depersonalization } \\
\text { Group }\end{array}$} & \multirow[b]{2}{*}{ total } \\
\hline & & $\mathrm{n}$ & $(\%)$ & $\mathrm{n}$ & $(\%)$ & $\mathrm{n}$ & $(\%)$ & $\mathrm{n}$ & $(\%)$ & \\
\hline All & Age 20 and above & 174 & $(28.5)$ & 145 & $(23.8)$ & 46 & $(7.5)$ & 245 & $(40.2)$ & 610 \\
\hline \multirow[t]{2}{*}{$(\mathrm{n}=1461)$} & Age 30 and above & 161 & $(35.6)$ & 87 & $(19.2)$ & 42 & $(9.3)$ & 162 & $(35.8)$ & 452 \\
\hline & Age 40 and above & 180 & $(45.1)$ & 71 & $(17.8)$ & 41 & $(10.3)$ & 107 & $(26.8)$ & 399 \\
\hline Major city & Age 20 and above & 36 & $(30.8)$ & 24 & $(20.5)$ & 6 & (5.1) & 51 & $(43.6)$ & 117 \\
\hline \multirow[t]{2}{*}{$(\mathrm{n}=1074)$} & Age 30 and above & 52 & $(44.8)$ & 21 & $(18.1)$ & 13 & $(11.2)$ & 30 & $(25.9)$ & 116 \\
\hline & Age 40 and above & 63 & $(40.9)$ & 26 & $(16.9)$ & 14 & $(9.1)$ & 51 & $(33.1)$ & 154 \\
\hline Regional city & Age 20 and above & 138 & $(28.0)$ & 121 & $(24.5)$ & 40 & $(8.1)$ & 194 & $(39.4)$ & 493 \\
\hline \multirow[t]{2}{*}{$(\mathrm{n}=387)$} & Age 30 and above & 109 & $(32.4)$ & 66 & (19.6) & 29 & (8.6) & 132 & $(39.3)$ & 336 \\
\hline & Age 40 and above & 117 & $(47.8)$ & 45 & $(18.4)$ & 27 & $(11.0)$ & 56 & $(22.9)$ & 245 \\
\hline
\end{tabular}

Analysis of the relation of burnout score to the other variables indicated that age group (twenties and thirties), married, raising children, high stress score, and the 3 coping sub-scales were significantly correlated ( $\mathrm{p}<0.001)$. There was no correlation with outpatient care, number of overtime hours, or work schedule (including night shift).

\subsection{Relation between Burnout Categories and Nurse-Related Factors}

We performed multiple logistic regression analysis with the 4 types of burnout as the dependent variables and the items found to have a significant relation to burnout (nurses' attributes, coping, and stress score) as the independent va- 
riables, and we divided the results by age group. The results indicated that, when we used the Burnout Low Group as a reference category, being in the twenties age group (odds ratio 2.073, $\mathrm{p}=0.001$ ) and the stress score (odds ratio 1.461, $\mathrm{p}<$ 0.000) had an effect in the Emotion Group, while childcare (odds ratio 0.517), emotionally-focused behaviors related to coping behaviors (odds ratio $0.767, \mathrm{p}<$ 0.000 ), and avoidance behaviors (odds ratio 1.190, p < 0.000) had an effect in the Depersonalization Group regardless of the age group of the subject.

In the Emotion/Depersonalization Group, the influential factors were being in the twenties age group (odds ratio 1.982, $\mathrm{p}=0.002$ ), being in the thirties age group (odds ratio 1.720, $\mathrm{p}=0.010$ ), childcare (odds ratio 0.497, $\mathrm{p}<0.000$ ), and stress score (odds ratio 1.889, $\mathrm{p}<0.000$; Table 4).

Table 4. Relation between the 4 types of Burnout and personal factors by age group (multiple logistic regression analysis).

\begin{tabular}{|c|c|c|c|c|c|c|c|c|c|c|c|}
\hline \multicolumn{12}{|c|}{$\mathrm{N}=1461$} \\
\hline \multicolumn{2}{|c|}{4 Types of Burnout } & \multirow[t]{2}{*}{ Factor } & \multirow[t]{2}{*}{$\begin{array}{l}\text { Odds } \\
\text { ratio }\end{array}$} & \multirow{2}{*}{$\begin{array}{c}95 \% \\
\text { Confidence } \\
\text { Interval }\end{array}$} & \multirow[t]{2}{*}{ p-value } & \multirow[t]{2}{*}{$\begin{array}{l}\text { Odds } \\
\text { ratio }\end{array}$} & $\begin{array}{c}95 \% \\
\text { Confidence } \\
\text { Interval }\end{array}$ & \multirow[t]{2}{*}{$\mathrm{p}$-value } & $\begin{array}{l}\text { Odds } \\
\text { ratio }\end{array}$ & $\begin{array}{c}95 \% \\
\text { Confidence } \\
\text { Interval }\end{array}$ & \multirow[t]{2}{*}{ p-value } \\
\hline & & & & & & & $30 \mathrm{~s}(\mathrm{n}=452)$ & & & $\mathrm{s}$ and older $(\mathrm{n}=$ & \\
\hline \multirow{7}{*}{$\begin{array}{l}\text { Emotion High } \\
\text { Group }\end{array}$} & Personal & Spouse & 3.114 & $1.288-7.526$ & 0.012 & 1.081 & $0.501-2.334$ & 0.842 & 0.683 & $0.332-1.405$ & 0.300 \\
\hline & attributes & Children & 0.235 & $0.057-0.964$ & 0.044 & 0.652 & $0.308-1.381$ & 0.264 & 1.009 & $0.489-2.083$ & 0.981 \\
\hline & & Night shift & 1.222 & $0.494-3.027$ & 0.664 & 0.826 & $0.426-1.604$ & 0.573 & 0.570 & $0.294-1.105$ & 0.096 \\
\hline & Coping & Problem-focused & 1.070 & $0.974-1.175$ & 0.158 & 1.002 & $0.894-1.122$ & 0.977 & 1.008 & $0.899-1.130$ & 0.893 \\
\hline & & Emotion-focused & 0.918 & $0.797-1.056$ & 0.232 & 0.977 & $0.832-1.147$ & 0.775 & 0.982 & $0.816-1.182$ & 0.848 \\
\hline & & Avoidance-focused & 1.026 & $0.944-1.115$ & 0.553 & 1.023 & $0.929-1.126$ & 0.648 & 1.020 & $0.921-1.130$ & 0.699 \\
\hline & Stress & Stress score & 1.521 & $1.363-1.696$ & 0.000 & 1.411 & $1.252-1.590$ & 0.000 & 1.472 & $1.295-1.674$ & 0.000 \\
\hline \multirow{7}{*}{$\begin{array}{c}\text { Depersonalization } \\
\text { Group }\end{array}$} & Personal & Spouse & 4.171 & $1.467-11.862$ & 0.007 & 0.675 & $0.250-1.824$ & 0.438 & 0.945 & $0.397-2.246$ & 0.898 \\
\hline & attributes & Children & 0.924 & $0.228-3.737$ & 0.912 & .503 & $0.188-1.343$ & 0.170 & 0.539 & $0.224-1.296$ & 0.167 \\
\hline & & Night shift & 0.702 & $0.244-2.020$ & 0.512 & 1.198 & $0.460-3.125$ & 0.711 & 1.074 & $0.469-2.460$ & 0.867 \\
\hline & Coping & Problem-focused & 1.080 & $0.943-1.237$ & 0.268 & 0.991 & $0.859-1.144$ & 0.902 & 1.058 & $0.923-1.213$ & 0.417 \\
\hline & & Emotion-focused & 0.742 & $0.608-0.906$ & 0.003 & 0.837 & $0.685-1.023$ & 0.082 & 0.746 & $0.600-0.928$ & 0.009 \\
\hline & & Avoidance-focused & 1.227 & $1.092-1.379$ & 0.001 & 1.141 & $1.006-1.295$ & 0.040 & 1.190 & $1.055-1.343$ & 0.005 \\
\hline & Stress & Stress score & 1.250 & $1.075-1.453$ & 0.004 & 1.191 & $1.025-1.384$ & 0.022 & 1.376 & $1.190-1.591$ & 0.000 \\
\hline \multirow{7}{*}{$\begin{array}{c}\text { Emotion/ } \\
\text { Depersonalization } \\
\text { Group }\end{array}$} & Personal & Spouse & 3.661 & $1.475-9.084$ & 0.005 & 0.821 & $0.392-1.720$ & 0.601 & 0.572 & $0.278-1.175$ & 0.128 \\
\hline & factors & Children & 0.371 & $0.097-1.425$ & 0.149 & 0.414 & $0.199-0.860$ & 0.018 & 0.683 & $0.332-1.405$ & 0.301 \\
\hline & & Night shift & 1.536 & $0.611-3.858$ & 0.361 & 0.569 & $0.300-1.081$ & 0.085 & 0.499 & $0.259-0.964$ & 0.039 \\
\hline & Coping & Problem-focused & 1.072 & $0.973-1.180$ & 0.158 & 0.946 & $0.849-1.054$ & 0.312 & 1.017 & $0.909-1.138$ & 0.767 \\
\hline & & Emotion-focused & 0.848 & $0.735-0.979$ & 0.025 & 0.917 & $0.788-1.067$ & 0.264 & 0.872 & $0.728-1.045$ & 0.138 \\
\hline & & Avoidance-focused & 1.144 & $1.051-1.244$ & 0.002 & 1.123 & $1.024-1.231$ & 0.014 & 1.152 & $1.040-1.276$ & 0.006 \\
\hline & Stress & Stress score & 2.129 & $1.883-2.407$ & 0.000 & 1.690 & $1.502-1.900$ & 0.000 & 1.874 & $1.635-2.148$ & 0.000 \\
\hline
\end{tabular}

The Low Group was used as the reference category. The age groups were 20s, 30s, and 40s and older. Stress score: Total score of 11 items that indicate the state of physical and mental stress (No appetite, become excited, have anxiety, feel listless, feel blue, feel bothersome, cannot calm down, cannot sleep, feel severely tired, feel exhausted, feel depressed). Score range: 0 - 11 points. 


\subsection{Relation of Nurse-Related Factors by Burnout Category and Age Group (Results of Multiple Regression Analysis)}

Next, we performed multiple logistic regression analysis with the 4 burnout categories by age group as the dependent variables and the items that were found to have a significant correlation to burnout (nurses' attributes, coping, stress score) as the independent variables. The results indicated that, when the Burnout Low Group was used as a reference category, marital status (odds ratio 3.114, $\mathrm{p}=$ 0.012 ) and childcare (odds ratio $0.235, \mathrm{p}=0.044$ ) only in the twenties age group had an effect in the Emotion Group, and that the stress score had a strong effect regardless of the age group (odds ratio $1.411-1.521, \mathrm{p}=0.000$ ). Coping was not found to have had an effect. In the Depersonalization Group, marital status (odds ratio 4.171, $\mathrm{p}=0.007$ ) was significant only for those in the twenties age group. No other personal attributes were found to be related. Our analysis of coping indicated that although emotion-focused behaviors (odds ratio $0.742, \mathrm{p}=$ 0.003 ) were related only in the twenties age group, avoidance behaviors were related to all age groups (odds ratio $1.141-1.227, \mathrm{p}=0.001-0.040$ ). Stress score was a strong influential factor for all age groups (odds ratio 1.191 $-1.376, \mathrm{P}=$ 0.000 - 0.02). In the Emotion/Depersonalization Group, being married (odds ratio 3.661, $\mathrm{p}=0.005$ ) had an effect on those in the twenties age group, childcare (odds ratio $0.414, \mathrm{p}=0.018$ ) had an effect on those in the thirties age group, and night shift (odds ratio $0.499, \mathrm{p}=0.039$ ) had an effect on those in the forties age group. Analysis of coping indicated that emotion-focused behaviors (odds ratio $0.848, p=0.025$ ) had an effect only on those in the twenties age group, while avoidance had an effect on all age groups (odds ratio $1.123-1.152, \mathrm{p}=0.002$ 0.014 ). Stress score was an influential factor for all age groups (odds ratio 1.690 2.129, $\mathrm{p}<0.000$; Table 4).

\section{Discussion}

We were able to elucidate the state of burnout among nurses employed in hospitals in regional cities in Hokkaido.

\subsection{Characteristics of the Subjects}

The nurses who were the subjects of this study included more who were in younger age groups as compared to the statistics indicated in the nurses' employment survey conducted by the Ministry of Health, Labour and Welfare in 2015 (twenties $=22 \%$, thirties $=31 \%$, forties and older $=47 \%)[1]$. Ninety percent of the nurses in their twenties were employed at hospitals. The hospitals that participated in this study were regional cancer treatment core hospitals with a high number of beds, and we believe that the fact that the nurses were comparatively young was reflected in the results of this study. In comparison to the average monthly overtime hours reported by the Japanese Nursing Association (23.4 hours, 2008) [2], 84.7\% of the nurses in this study worked fewer than 20 hours of overtime per month, which showed a trend toward fewer overtime hours. In comparison to the mean number of paid vacation days taken by nurses 
as reported abovementioned survey by the Japan Nursing Association (8.4 days) [2], $65.8 \%$ of the nurses in this study took fewer than 10 days and $31.6 \%$ took 10 - 20 days, indicating that younger nurses were in environments that allowed them to take a relatively high number of paid vacation days.

\subsection{State of Burnout among Nurses}

In comparison to the results of the survey of nurses at hospitals with at least 800 beds conducted by Ogata et al. (emotional exhaustion, depersonalization, sense of personal accomplishment $=3.6,2.3,2.5$ ) [13], the burnout scores for the subjects in the present study indicated that sense of personal accomplishment was higher while emotional exhaustion and depersonalization were the same. We believe that since the basic attributes, such as the relatively young age groups the nurses belonged to, were similar, comparison of these two studies is valid, and therefore the results reflect the tendency for nurses with less experience to have more emotional exhaustion. Although there are reports indicating that burnout is related to the number of years of nursing experience, when we looked at the data for our 4 burnout groups we found that $35 \%$ - $40 \%$ of those in their twenties and thirties were in the Emotion Group and Emotion/Depersonalization Group, which we believe indicates that developing strategies designed to help less experienced nurses and mid-career nurses cope with burnout is an urgent issue. While researchers have a variety of interpretations of the structure and process of burnout [7] [8] [9], most of the subjects of the present study were in the Emotion/Depersonalization Group followed by the Emotion Group, which indicates fewer subjects were in the group in which only depersonalization was high. There were a particularly high number of nurses in their thirties who worked at regional hospitals in the Emotion/Depersonalization Group. Based on the fact that there were no differences between the amount of night shift work and number of overtime hours worked in major cities and regional cities, we assume that stress-related factors include personal relations at the workplace, heavy workload, and conflict with household responsibilities [14]. There is a possibility that physical and mental and physical fatigue and sleep difficulties of local medical workers, which are caused in association with various factors such as shortage of human resources, may be influential on the reason why hospital nurses working in local cities tend to burn out more than urban areas [15]. Shift work is one characteristic of nursing work, and although it is said to be a factor related to job separation [3], in the present study we did not detect a relation between burnout and night shift work among 20s and 30s nurses. In recent years, consideration has been paid to nurses coming back to work after childcare leave, including exempting them from night shift work, rotating their work schedules only among day-shift positions, and shortening their overall work hours [16]. This has led to nurses feeling that night shift work was less of a burden, and this may have decreased the influence of night shift work. Since we did not ascertain the number of times the night shift was worked during the one-month period of this study, the qualitative relation of the number of times 
shift work is scheduled remains a topic for future study. In a previous study the presence of a spouse was reported to decrease emotional exhaustion and increase depersonalization. In the present study, these were factors in all three Groups (Emotion, Depersonalization, and Emotion/Depersonalization) only among those in their twenties. We assume this result was due to the fact that nurses in their twenties are taking on household roles as a result of life events such as marriage, which makes it increasingly difficult to balance these responsibilities with their clinical responsibilities as nurses, which in turn leads to conflict. We also assume that this result supports the notion that marriage is a factor related to job separation among nurses in their twenties. On the other hand, our investigation of childcare indicated that regardless of whether the subjects worked the night shift or not, there were fewer numbers of nurses in their twenties in the Emotion Group and fewer numbers of nurses in their thirties in the Emotion/Depersonalization Group. We believe that this may be due to the fact that childcare does not simply create a burden in the household, it also has a positive influence on nurses' emotional stability and stress management skills. Nurses engaged in childcare themselves recognize that raising children is a factor related to continuing to work and is effective in raising their awareness of the importance of stress management.

Analysis of coping behaviors indicated that those of all ages in the Depersonalization Group were affected by avoidance behaviors. Depersonalization is thought to be a coping behavior that arises as a result of emotional exhaustion [10], and therefore we believe that among those in the Depersonalization Group there was a correlation to the avoidance behavior of not paying attention to those around them, which resulted from a depletion of emotional energy. We found a correlation between those in their twenties who were in the Depersonalization Group and the Emotion/Depersonalization Group and emotion-focused behaviors. However, this may also indicate that the nurses were simply unable to successfully perform "emotion-adjusting behaviors" as a type of stress management. In addition, problem-solving-focused behaviors were not found to be an influential factor for the subjects of the present study. Based on the fact that a previous study [4] reported that when supportive resources are available, coping in the form of problem-solving reduces the stress response, we believe it is necessary to investigate coping behaviors in conjunction with the availability of supportive resources. In particular, there is a need for those in their twenties, who are prone to experiencing high levels of stress, to be more aware of their own coping behaviors and receive support in the form of education regarding effective ways of managing stress. In this study, the stress score used in this study to ascertain the state of physical and mental health consisted of a workplace stress check questionnaire with 11 assessment items. In this study, the stress score had a strong influence over subjects of all ages who were in all the Burnout groups. In particular, we believe that they can be used as powerful predictive factors for those in the Emotion/Depersonalization Group and the Emotion Group. 


\subsection{Recommendation}

The stress scores used for this study are based on items that examine the degree of physical and psychological fatigue. A high stress score is considered to be related to nurse burnout or, in other words, a strong indicator of burnout prediction. Since 2015 it has become mandatory for workplaces with at least $50 \mathrm{em}$ ployees to conduct workplace stress checks, and therefore many hospitals are conducting workplace stress checks for their nursing staff. The items on our questionnaire that were related to physical and mental stress responses can be used not only for health management but also in order to allow nurses themselves to become more aware of indications that may predict the onset of burnout. Therefore, we believe these questionnaire items are useful in both workplace management and health management. However, since nurses can experience burnout even with a stress score of 6 or less, it is believed that there are multiple factors influencing burnout, some of which are exclusive of psychosomatic stress symptoms. Therefore, it is necessary to educate nurses in their 20s on emotional coping strategies. Moreover, although it is not necessary to have a spouse risk the occurrence of burnout, it is important to consider ways to balance family life and work, taking into account career development as well. It was suggested that efforts geared toward work-life balance for nurses in their 20s and 30s are important. Nurses who are raising children are likely to face difficulties in balancing their nursing career with family life. Restricting night-shift work and decreasing working hours can help prevent burnout among such nurses. Thus, it is necessary to promote a daily work style that supports child rearing. In the case of nurses in their 40s, fatigue may increase due to day-shift work where the workload increases. I think that it is necessary to assign shift work while being mindful of the individual's workload and the negative impact of night shifts. An appropriate amount of night shifts can ease the pressure. Along with the stress score, it was shown that nurses' age, whether or not they engaged in child-rearing, and their coping styles are individual factors related to burnout. From this, we consider the complex relationship between stress score and other factors in nurse burnout prediction and examine an algorithm related to burnout.

\subsection{Limitations and Problems with This Study}

This study was a cross-sectional study targeting 4 relatively large-scale facilities in Hokkaido, and as a result its representativeness of the nursing population as a whole is limited. In addition, we investigated burnout and related factors of nurses who are employed in hospitals by age group and state of burnout. We did not investigate other factors such as workplace environment or human relations in this study. Therefore, it remains necessary to verify a burnout predictive index as well as nurses' desire to leave their jobs.

\section{Conclusion}

Analysis of the nurses who are employed at hospitals and who are at high risk of 
burnout due to a high level of emotional exhaustion which causes depersonalization indicated that $40.2 \%$ were in their twenties, $35.8 \%$ were in their thirties, and $26.8 \%$ were in their forties. The factors related to the burnout state differed according to the nurses' age groups. The factors found to be related to burnout were marital status and childcare for nurses in their twenties, and childcare for nurses in their thirties. Coping behaviors were not influenced by problem-focused behaviors, but non-emotion-focused behaviors and avoidance-focused behaviors were found to be influential factors. It was found that stress score, which indicates physical and mental stress responses, was a factor that influenced all burnout types for all age groups, and in particular could be used as a predictive index for those who had high scores for both emotional exhaustion and depersonalization.

\section{Acknowledgements}

The authors would like to express their appreciation for the kind cooperation of all the nurses who participated in this study.

\section{Conflicts of Interest}

The authors declare that there were no conflicts of interest to report.

\section{Research Funding}

This study was partially funded by a Ministry of Public Management, Home Affairs, Posts and Telecommunications SCOPE regional ICT promotion research and development grant (152301001) and was conducted as an empirical study for use in cloud services and the development of ICT for the purpose of the health management of nurses and the improvement of regional medical care.

\section{References}

[1] Japanese Ministry of Health, Labor and Welfare (2015) Number of Nurses, Public Health Nurses, Associate Nurses. http://www.mhlw.go.jp/toukei/saikin/hw/eisei/14/dl/kekka1.pdf

[2] Japan Nursing Association (2013) Overtime among Hospital Nurses in Japan. https://www.nurse.or.jp/nursing/shuroanzen/jikan/pdf/20090427.pdf

[3] Motomura, Y. and Yatsushiro, R. (2010) Factors Relating to Nurse Burnout. Japanese Society of Occupational Medicine and Traumatology, 58, 120-127. (In Japanese)

[4] Inaba, R. and Inoue, M. (2001) Relationship between Burnout and Work-Related Stress among Female Nurses with a Special Scope to Occupational Career. Japanese Society of Occupational Medicine and Traumatology, 59, 129-136. (In Japanese)

[5] Maslach, C. (1981) The Measurement of Experienced Burnout. Journal of Occupational Behavior, 2, 99-113. https://doi.org/10.1002/job.4030020205

[6] Kubo, M. (2007) Burnout Syndrome-The Stress among Human Services Professionals. The Monthly Journal of the Japan Institute of Labour, 558, 54-64.

[7] Kitaoka-Higashiguchi, K. and Nakagawa, H. (2003) Job Strain, Coping, and Burnout among Japanese Nurses. Japanese Journal of Health and Human Ecology, 69, 
66-79.

[8] Shimizu, T., Feng, Q. and Nagata, S. (2005) Relationship between Turnover and Burnout among Japanese Hospital Nurse. Journal of Occupational Health, 47, 334-336. ( In Japanese)

[9] Neville, K. and Cole, D. (2013) The Relationship among Health Promotion Behavior, Compassion Fatigue, Burnout, and Compassion Satisfaction in Nurses Practicing in a Community Medical Center. Journal of Nursing Administration, 43, 348-354. https://doi.org/10.1097/NNA.0b013e3182942c23

[10] Japanese Ministry of Industry Safe and Health Act (2015) The Brief Job Stress Questionnaire. http://www.mhlw.go.jp/bunya/roudoukijun/anzeneisei12/

[11] Japanese Nursing Association (2013) Nurse's Guideline on Night Shift and Shift Work. https://www.nurse.or.jp/nursing/shuroanzen/yakinkotai/guideline/index.htm

[12] Ozeki, Y. (1993) Refining the Stress Self-Rating Scale for University Students-Toward a Transactional Analysis. The Annual of the Graduate School of Comparative Studies of International Cultures and Societies, 1, 95-114.

[13] Ogata, Y. and Nagano, M. (2012) The Relationship between Burnout and Work Environment among Hospital Nurses in Japan. Journal of Graduate School of Nursing, Chiba University, 34, 39-44. (In Japanese)

[14] Ribeiro, V., Filho, F., Valenti, V., et al. (2014) Prevalence of Burnout Syndrome in Clinical Nurses at a Hospital of Excellence. International Archives of Medicine, 7, 22.

[15] Sumi, N., Sugimura, N., Yoshida, Y., et al. (2017) The State of Fatigue and Sleep among Clinical Nurses in Japan. Open Journal of Nursing, 7, Article ID: 81377. https://doi.org/10.4236/ojn.2017.712104

[16] Kagamiyama, H. and Yano, R. (2017) A Literature Review of Sleep among Nurses Doing Shift Work. Journal of Comprehensive Nursing Research, 18, 3-11. (In Japanese) 